\title{
Sección: Política Internacional
}

\section{Dra. Lía Rodríguez de la Vega}

Coordinadora de Sección

\section{Introducción}

La globalización es un proceso, que se presenta como un fenómeno diferente de la mundialización, que como ella implica modificaciones centrales en lo que hace al espacio y al tiempo, en su caso, en términos de ubicuidad e instantaneidad en ámbitos clave de la actividad social -asociadas a la creciente interconexión e interdependencia- (del Arenal Moyúa, 2008) y que por tal, representa un "punto de discontinuidad histórica" (Castells, 1997, p. 94).

Estos cambios pronunciados, exigen revisitar otras consideraciones acerca del poder y su ejercicio. En este sentido, Nye establece una diferencia entre distintos tipos de poder. Considera al poder duro como "la capacidad de lograr que los demás actúen de forma contraria a sus preferencias y estrategias iniciales" (Nye, 2011, p.11). Ello implica la capacidad de coaccionar, por medio de amenazas e incentivos. Por su parte, el poder blando es considerado "la capacidad de alcanzar objetivos mediante la atracción en lugar de la coacción" (Nye, 2004, X)1. "Surge del atractivo de la cultura de un país, de sus ideales políticos y de sus políticas" (Nye, 2004, X). En línea con ello, McMillan (2018) señala que el poder blando funciona indirectamente cuando es independiente del control del gobierno (cultura popular) y directamente a través de la diplomacia cultural, de modo que los actores de la política exterior actúan como canales de ese poder.

En ese marco, se produce la internacionalización de la educación superior, definida por la Asociación Internacional de Universidades como un proceso que integra una dimensión o perspectiva internacional o intercultural en las funciones principales de las universidades (enseñanza, investigación y servicios). Como bien señala Negrín Reyes (2015), se trata de un proceso manifiesto hacia el interior de la institución, al tiempo que es también una estrategia para la difusión tanto de su oferta educativa como de investigación, en el marco de un espacio supranacional. Al

\footnotetext{
${ }^{1}$ Finalmente, el autor introduce la idea del poder inteligente, entendido como el "equilibrio del poder duro y blando" (Nye, 2004, p. 32).
} 
respecto es conocido que el sistema de educación superior de la India sigue contribuyendo a la creación de capacidades en diversas regiones de Asia y África².

Así, el paso de la India a ser una nación implicada en la cooperación sur-sur, norte sur y cooperación triangular, es vista como efecto de la creciente globalización, al tiempo que de la internacionalización de la educación en el planeta (Khare, 2015). En línea con lo señalado, en 1964, India instituyó el Programa de Cooperación Económica y Técnica, como un programa de asistencia bilateral del gobierno de la India. El programa, que abarca más de 160 países de Asia y el Pacífico, África, América Latina y el Caribe y Europa del Este y Central, posee diversas aristas, de las cuales la de entrenamiento y construcción de capacidades es una de las más importantes (Indian Technical \& Economic Cooperation Programme, Ministry of External Affairs, Government of India, 2015).

Por otro lado, las matrices culturales que se ponen en juego en la interacción de sujetos, grupos, otros agentes sociales y países, suponen también perspectivas específicas acerca del espacio, el tiempo y el medioambiente (en términos del lugar de la propia especie humana en el planeta, el tipo de uso de los recursos naturales, los modelos de desarrollo adoptados, etc.), que han implicado e implican acciones conjuntas de los diversos países (con un rol destacado de China e India en ello, en tanto hacen grandes demandas de energía y al mismo tiempo, avanzan en extender iniciativas como por ejemplo, aquellas asociadas a energías $\operatorname{limpias}^{3}$ ).

Bloch (2015) apunta que desde la década de los '90 la temática de los recursos naturales estratégicos (petróleo y gas, agua dulce, biodiversidad, minerales estratégicos, etc.) viene cobrando relevancia en las relaciones internacionales. Destaca que resultan estratégicos porque tanto los estados como las empresas buscan obtenerlos por razones securitarias, con un correlato existente en la atención del aprovechamiento y protección de tales recursos. Cabe sumar a esto la condición

\footnotetext{
2 El gobierno indio, SAARC mediante, proclamó una política de cooperación regional en educación superior para lograr desarrollar las capacidades en la zona, operacionalizándose la agenda propuesta por medio de un Comité de Jefes de la Comisión de Becas Universitarias / Organismos equivalentes (Yeravdekar y Tiwari, 2014).

${ }^{3} \mathrm{~A}$ modo de ejemplo, puede decirse que el Primer Ministro de la India, Narendra Modi, se ha propuesto alcanzar 100 GW (gigavatios) de energía solar para 2022, del total de $175 \mathrm{GW}$, que India planea producir a partir de fuentes renovables. Esto fue anunciado en la Cumbre de la Alianza Solar Internacional, celebrada en India, este año (Basu, 2018). En el caso de China, la Agencia Internacional de Energía (AIE), anunció que el país instaló más de 34 gigavatios de capacidad solar en 2016, duplicando lo hecho en Estados Unidos y esa cifra constituye casi la mitad de la capacidad total agregada en todo el mundo ese año. Este impresionante desempeño tiene otras aristas, tales como la de la granja solar más grande del mundo (el Parque Solar Longyangxia Dam, con sus 30 $\mathrm{km}$ cuadrados) y la granja solar flotante más grande del mundo, que fuera abierta hace poco tiempo, en Huainan, provincia de Anhui (Baraniuk, 2017).
} 
de finitud de tales recursos, en razón de lo cual, la reducción de los mismos, los costos crecientes de su explotación, el crecimiento de la población y los fenómenos asociados al cambio climático, contribuyen a estimular las tensiones ya existentes sobre el tema.

Sostiene de esta manera que se estaría configurando una nueva geografía de conflictos o de cooperación en el mundo, delimitada por la existencia, producción, comercialización y consumo de esos recursos naturales estratégicos.

Finalmente, ambas temáticas abordadas en este número del Journal de Ciencias Sociales, consideran casos relativos a Asia, aunque las reflexiones pueden hacerse extensivas a otros contextos. Las autoras son: Sabrina Victoria Olivera, abogada y miembro del Grupo de Trabajo sobre India y Asia del Sur, del Comité de Asuntos Asiáticos del Consejo Argentino para las Relaciones Internacionales (C.A.R.I.) y Mercedes Solé, Licenciada en Periodismo y miembro del mismo Grupo de Trabajo del C.A.R.I.

\section{Referencias}

Baraniuk, C. (2017). Future Energy: China leads world in solar power production. BBC News (22 de junio). Recuperado de http://www.bbc.com/news/business-40341833

Basu, N. (2018). India will generate $100 \mathrm{GW}$ of solar power by 2022, says Modi. The Hindu Business Line (11 de marzo). Recuperado de https://www.thehindubusinessline.com/economy/india-will-generate-100-gw-ofsolar-power-by-2022-says-modi/article23042063.ece

Bloch, R. (2015). Recursos naturales estratégicos: una "nueva geografía" se despliega. Voces en el Fénix, 50 "lluminados por el Fuego. Un mundo en Guerra". Recuperado de http://www.vocesenelfenix.com/content/recursos-naturalesestrat\%C3\%A9gicos-una-\%E2\%80\%9Cnueva-geograf\%C3\%ADa\%E2\%80\%9Dse-despliega

Castells, Manuel (1997). La era de la información. Economía, sociedad y cultura. Vol. 1: La sociedad red. Madrid, Alianza Editorial.

Del Arenal Moyúa, C. (2008). Mundialización, creciente interdependencia y globalización en las relaciones internacionales. Cursos de derecho internacional y relaciones internacionales de Vitoria-Gasteiz, 1. Recuperado de http://www.ehu.eus/cursosderechointernacionalvitoria/ponencias/pdf/2008/2008_ 4.pdf 
Indian Technical \& Economic Cooperation Programme, Ministry of External Affairs, Government of India (2015). Disponible en https://www.itecgoi.in/about.php Khare, M. (2015). India's Emergence as a Regional Education Hub. International Higher Education, 83, 26-26. Recuperado de file:///C:/Users/user/Downloads/908815502-1-SM.pdf

McMillan, M. (2018). La nueva plataforma de diplomacia cultural de la UE: Un medio de "poder blando" en un mundo multipolar. Global Affairs. Strategic Studies. Facultad de Derecho-relaciones Internacionales, Universidad de Navarra. Recuperado de http://www.unav.edu/web/global-affairs/detalle//blogs/16890541?_33_redirect=http\%3A\%2F\%2Fwww.unav.edu\%2Fweb\%2Fglo bal-

affairs\%2Fdetalle\%3Fp_p_id\%3D33\%26p_p_lifecycle\%3D0\%26p_p_state\%3Dn ormal\%26p_p_mode\%3Dview\%26p_p_col_id\%3Dcolumn1\%26p_p_col_count\%3D1\%26_33_struts_action\%3D\%252Fblogs\%252Fview

Negrín Reyes, D. E. (2015). Internacionalización de la educación superior: las relaciones internacionales académicas en la Universidad de Ciencias Médicas de Pinar del Río. Rev Ciencias Médicas Pinar del Río, 19 (5), 911-925. Recuperado de http://scielo.sld.cu/pdf/rpr/v19n5/rpr14515.pdf

Yeravdekar, V. R. y Tiwari, G. (2014). Internationalization of Higher Education in India: Contribution to Regional Capacity Building in Neighbouring Countries. Procedia Social and Behavioral Sciences 157, 373 - 380. Recuperado de https://ac.elscdn.com/S1877042814058625/1-s2.0-S1877042814058625main.pdf?_tid=e558336b-a53a-4484-ab21dc12974cfe24\&acdnat=1524540444_398d6cade11 efdd52731b366ddecbb02 